\title{
Weak Minimal Area in Entanglement Entropy
}

\author{
Shesansu Sekhar Pal ${ }^{1,2}$ and Shubhalaxmi Rath ${ }^{2}$ \\ ${ }^{1}$ Department of Physics, Utkal University, Bhubaneswar 751004, India \\ ${ }^{2}$ Centre of Excellence in Theoretical and Mathematical Sciences, Siksha 'O' Anusandhan University, \\ Khandagiri Square, Bhubaneswar 751030, India
}

Correspondence should be addressed to Shesansu Sekhar Pal; shesansu@gmail.com

Received 18 November 2014; Accepted 8 February 2015

Academic Editor: Sally Seidel

Copyright (C) 2015 S. S. Pal and S. Rath. This is an open access article distributed under the Creative Commons Attribution License, which permits unrestricted use, distribution, and reproduction in any medium, provided the original work is properly cited. The publication of this article was funded by SCOAP ${ }^{3}$.

\begin{abstract}
We revisit the minimal area condition of Ryu-Takayanagi in the holographic calculation of the entanglement entropy, in particular, the Legendre test and the Jacobi test. The necessary condition for the weak minimality is checked via Legendre test and its sufficient nature via Jacobi test. We show for AdS black hole with a strip type entangling region that it is this minimality condition that makes the hypersurface unable to cross the horizon, which is in agreement with that studied earlier by Engelhardt et al. and Hubeny using a different approach. Moreover, demanding the weak minimality condition on the entanglement entropy functional with the higher derivative term puts a constraint on the Gauss-Bonnet coupling; that is, there should be an upper bound on the value of the coupling, $\lambda_{a}<(d-3) / 4(d-1)$.
\end{abstract}

\section{Introduction}

The recent conjecture on the holographic formulation of the entanglement entropy by Ryu-Takayanagi (RT) $[1,2]$ has given a new direction to do explicit calculations in the field theory provided that it admits a dual gravitational description (in a recent development in [3], the authors have conjectured the existence of a geometric entropy in a theory of quantum gravity that includes it in the entanglement entropy) [4-7]. In order to compute the entanglement entropy of a given region, $A$, with its complement in the field theory, it proposes with a fixed time slice to consider a codimension two-hypersurface, $\Sigma$, in the bulk in such a way that its boundary coincides with the boundary of the region under study; that is, $\partial A=$ $\partial \Sigma$. Moreover, we need to consider the hypersurface that minimizes the area. In this case, the entanglement entropy is simply given by the area of the hypersurface divided by $4 G_{N}$, where $G_{N}$ is Newton's constant and it reads as

$$
S_{E E}(A)=\lim _{\partial \Sigma=\partial A} \frac{\operatorname{Min}(\operatorname{Area}(\Sigma))}{4 G_{N}^{d+1}}
$$

Recall that the area of a codimension two-hypersurface is given by

$$
\begin{gathered}
\operatorname{Area}(\Sigma)=\int_{\Sigma} d^{d-1} \sigma \sqrt{\operatorname{det}\left(\partial_{a} X^{M} \partial_{b} X^{N} G_{M N}\right)}, \\
g_{a b} \equiv \partial_{a} X^{M} \partial_{b} X^{N} G_{M N},
\end{gathered}
$$

where $X^{M}$ and $G_{M N}$ are the embedding functions and the bulk geometry, respectively. Setting the first variation of such an area functional to zero gives the following equation which is essentially the equation of the hypersurface [8] and is further studied (some other interesting studies are reported in $[9,10])$ in $[11-14]$ :

$$
\begin{gathered}
g^{a b} \mathscr{K}_{a b}^{S}=0, \\
\mathscr{K}_{a b}^{S}=\partial_{a} \partial_{b} X^{S}-\gamma_{a b}^{c} \partial_{c} X^{S}+\partial_{a} X^{M} \partial_{b} X^{N} \Gamma_{M N}^{S},
\end{gathered}
$$

where $g^{a b}$ is the inverse of the induced metric, $g_{a b} \cdot \gamma_{a b}^{c}$ and $\Gamma_{M N}^{S}$ are the connections defined with respect to the induced metric on the hypersurface and the bulk geometry, respectively. 
In order to find the entanglement entropy, we can solve for $X^{M}$ 's in (3) for a given bulk geometry and substitute that into the area integral. However, it is not a priori clear that the solution of (3) will necessarily give us a minimum area. It can give a maximum, a minimum, or a point of inflection/saddle point. It is suggested in [15] that, by working with the Euclidean signature, the extremization of the area functional will automatically give a global minimum of the area functional. However, with the Minkowski signature, the extremization gives saddle points and one needs to opt for the solution that gives a minimum area.

In this paper, we want to study the (weak) minimal condition on the entanglement entropy functional with the Minkowski signature for generic $\Sigma$ that follows from (2) and study the consequences through some examples.

In order to check the minimality condition on the area or equivalently on the entanglement entropy functional, let us find the second variation of the area functional (2), which gives

$\delta^{2} \operatorname{Area}(\Sigma)$

$$
\begin{aligned}
=\int \sqrt{\operatorname{det} g_{a b}}[ & \left(\left(g^{a b} g^{c d}-2 g^{a c} g^{b d}\right)\right. \\
& \left.\cdot G_{K L} G_{M N} \partial_{b} X^{N} \partial_{d} X^{L}+g^{a c} G_{M K}\right) \\
\cdot \partial_{c} \delta X^{K} \partial_{a} \delta X^{M} & \\
+ & \left(\left(g^{a b} g^{c d}-2 g^{a c} g^{b d}\right) G_{K L} \partial_{P} G_{M N} \partial_{d}\right. \\
& \left.\cdot X^{L} \partial_{a} X^{M} \partial_{b} X^{N}+2 g^{b c} \partial_{b} X^{N} \partial_{P} G_{K N}\right) \\
& \cdot \partial_{c} \delta X^{K} \delta X^{P} \\
+ & \left(\frac{1}{4}\left(g^{a b} g^{c d}-2 g^{a c} g^{b d}\right) \partial_{a} X^{M} \partial_{b} X^{N} \partial_{P}\right. \\
& \cdot G_{M N} \partial_{c} X^{S} \partial_{d} X^{L} \partial_{K} G_{S L} \\
& \left.\left.+\frac{1}{2} g^{a b} \partial_{a} X^{M} \partial_{b} X^{N} \partial_{P} \partial_{K} G_{M N}\right) \delta X^{P} \delta X^{K}\right] \\
=\int V^{T} \cdot M \cdot V, &
\end{aligned}
$$

where the column vector $V=\left(\begin{array}{c}\partial \delta X \\ \delta X\end{array}\right)$ and we have dropped the indices, for simplicity. Note that, in getting the result, we have dropped a total derivative term, which essentially will give a boundary term, and we assume that it is not going to contribute to the boundary, also a term proportional to the equation of motion. If we want the area to be a minimum then the determinant of the matrix $M$ should be positive. The Jacobi test talks about the positivity of the matrix $M$ and it corresponds to the sufficient condition for the weak minimum.
In calculus, the Legendre test says that

$$
\begin{aligned}
\frac{\delta^{2}(\operatorname{Area}(\Sigma))}{\partial_{c} \delta X^{K} \partial_{a} \delta X^{M}} \\
=2 \sqrt{\operatorname{det}\left(g_{a b}\right)} \\
\quad \cdot\left[\left(g^{a b} g^{c d}-2 g^{a c} g^{b d}\right) G_{K L} G_{M N} \partial_{b} X^{N} \partial_{d} X^{L}+g^{a c} G_{M K}\right] \\
>0
\end{aligned}
$$

and it gives a weak condition on the minimality of the function, in this case the area. Generically, it is very difficult to combine (3) and (5) so as to draw any useful conclusion. (However, it is certainly very interesting to find connection between (5) and the extrinsic curvature as proposed in the context of black holes in [16], if there is any.) Instead, in what follows, we will calculate the quantity (5) in different examples and check whether the area is (weak) minimum or not.

In this paper, we study the consequence of such weak minimality condition in different spacetime, such as AdS spacetime with and without the black holes, hyperscale violating geometries, and geometries with higher derivative terms. In the case of the black hole geometry, the minimal area condition of the RT conjecture gives us a very interesting consequence; that is, the spacelike hypersurfaces do not cross the horizon. This conclusion matches precisely as studied in [17], where the author did not find any solution to the embedding field, $X^{M}$, of (3) inside the horizon and further studied in [12] at finite ' $t$ Hooft coupling and more generally in [16].

By studying different examples, we find that the second variation of the area functional can be written as

$$
\delta^{2} \operatorname{Area}(\Sigma)=\int \prod_{i} d x_{i}\left(A \delta r^{\prime} \delta r^{\prime}+B \delta r \delta r^{\prime}+C \delta r \delta r\right),
$$

where $r^{\prime}=d r / d x$ and $x$ is one of the coordinates on the hypersurface. The integral is over the world volume coordinates of the codimension two-hypersurface.

The weak minimality condition states that the second variation of the area functional with respect to $r^{\prime}$ becomes positive:

$$
A>0,
$$

and the Jacobi test says that the determinant of the matrix $M$ should be positive, $A C-B^{2} / 4>0$. In this paper, we will be checking these conditions by studying several examples.

It is also very interesting to ask the minimal nature of the entanglement entropy functional even in the finite 't Hooft coupling limit. (A prescription is given in $[18,19]$ to construct the entanglement entropy functional in such cases.) In this context, it is argued in $[18,20]$ based on the strong subadditivity property that the first possible higher derivative correction of the entanglement entropy functional indeed obeys the minimality condition. For our purpose, we 
consider the following entanglement entropy functional, as also studied in [21-23]:

$$
4 G_{N} S_{E E}=\int d^{d-1} \sigma \sqrt{\operatorname{det}\left(g_{a b}\right)}\left[1+\frac{2 \lambda_{a} R_{A}^{2}}{(d-2)(d-3)} R(g)\right] \text {, }
$$

where $R(g)$ denotes the Ricci scalar made out of the induced metric. We found the following constraint upon demanding the weak minimality of the entanglement entropy functional:

$$
\lambda_{a}<\frac{(d-3)}{4(d-1)}
$$

Note that we denote $R_{A}$ as the radius of the AdS spacetime. The constraint on the Gauss-Bonnet coupling, $\lambda_{a}$, does not coincide with the result obtained in $[24,25]$. So it means that the hypersurface under study does not have either minimal or maximal entanglement entropy. The maximal entanglement entropy is ruled out; otherwise, the GaussBonnet coupling can be as large as infinity. Through this study, there follows an important result; that is, theories without higher derivative terms do admit a minimal hypersurface but not with. (The caveat is that the weak minimality analysis is performed only to leading order in the coupling.) Hence, the nature of the hypersurface with the higher derivative term remains to be seen in the future.

\section{Example: Strip Type}

In this section, we will check the minimality of the area functional by doing some explicit calculation for the strip type entangling region. This will be performed by finding the embedding field that follows from (3). The strip on the field theory is defined as $0 \leq x_{1} \leq \ell$ and $-L / 2 \leq\left(x_{2}, \ldots, x_{d-1}\right) \leq$ $L / 2$. Moreover, the bulk geometry is assumed to take the following form:

$$
\begin{aligned}
d s_{d+1}^{2}= & -g_{t t}(r) d t^{2}+g_{x x}(r)\left(d x_{1}^{2}+\cdots+d x_{d-1}^{2}\right) \\
& +g_{r r}(r) d r^{2}
\end{aligned}
$$

With the following embedding fields $X^{t}=0, X^{a}=x^{a}=$ $\sigma^{a}, X^{r}=r\left(x_{1}\right)$, the induced metric is

$$
\begin{aligned}
& d s_{d-1}^{2}= g_{a b} d \sigma^{a} d \sigma^{b} \\
&= g_{x x}(r)\left(d x_{2}^{2}+\cdots+d x_{d-1}^{2}\right) \\
&+\left(g_{r r}(r) r^{\prime 2}+g_{x x}(r)\right) d x_{1}^{2}, \\
& r^{\prime} \equiv \frac{d r}{d x_{1}} .
\end{aligned}
$$

In this case, the area takes the following form: Area = $L^{d-2} \int d x_{1} g_{x x}^{(d-2) / 2} \sqrt{g_{x x}+g_{r r} r^{\prime 2}}$, whose second variation gives the following column vector, $V$, and the matrix, $M$ :

$$
M=\left(\begin{array}{cc}
A & \frac{B}{2} \\
\frac{B}{2} & C
\end{array}\right), \quad V=\left(\begin{array}{c}
\delta r^{\prime} \\
\delta r
\end{array}\right) .
$$

This means

$$
\begin{gathered}
\delta^{2} \text { Area }(\Sigma)=L^{d-2} \int\left(A \delta r^{\prime} \delta r^{\prime}+B \delta r \delta r^{\prime}+C \delta r \delta r\right) \\
=L^{d-2} \int A\left(\delta r^{\prime}+\frac{B}{2 A} \delta r\right)^{2} \\
+\frac{\left(4 A C-B^{2}\right)}{4 A} \delta r \delta r .
\end{gathered}
$$

In order to have a minimum area functional, $A$ should be positive and $4 A C>B^{2}$. Note that the determinant of the matrix $M$ is $\operatorname{det}(M)=A C-B^{2} / 4$ and $\delta^{2}(\operatorname{Area}(\Sigma)) / \delta r^{\prime} \delta r^{\prime} \sim$ $2 A$, where the expressions for these quantities are

$$
\begin{aligned}
A= & \frac{g_{x x}^{d / 2} g_{r r}}{\left(g_{x x}+r^{\prime 2} g_{r r}\right)^{3 / 2}}, \\
B= & g_{x x}^{(d-2) / 2} r^{\prime}\left(\frac{(d-2) g_{x x}^{\prime} g_{r r}+2 g_{r r}^{\prime}}{g_{x x} \sqrt{g_{x x}+r^{\prime 2} g_{r r}}}\right) \\
& -\frac{g_{x x}^{(d-2) / 2} g_{r r} r^{\prime}\left(g_{x x}^{\prime}+r^{\prime 2} g_{r r}^{\prime}\right)}{\left(g_{x x}+r^{\prime 2} g_{r r}\right)^{3 / 2}}, \\
C- & \frac{g_{x x}^{(d-2) / 2}\left(g_{x x}^{\prime}+r^{\prime 2} g_{r r}^{\prime}\right)^{2}}{4\left(g_{x x}+r^{\prime 2} g_{r r}\right)^{3 / 2}} \\
+ & \left(\frac{d-2}{4}\right) g_{x x}^{(d-6) / 2} \\
& \cdot \sqrt{g_{x x}+r^{\prime 2} g_{r r}}\left(g_{x x}^{\prime 2}(d-4)+2 g_{x x} g_{x x}^{\prime \prime}\right) \\
+ & \left(g_{x x}^{(d-2) / 2}\left(g_{x x}^{\prime \prime}+r^{\prime 2} g_{r r}^{\prime \prime}\right)\right. \\
& \left.+(d-2) g_{x x}^{(d-4) / 2} g_{x x}^{\prime}\left(g_{x x}^{\prime}+r^{\prime 2} g_{r r}^{\prime}\right)\right) \\
& \left(2 \sqrt{g_{x x}+r^{\prime 2} g_{r r}}\right)^{-1} \cdot
\end{aligned}
$$

The meaning of the derivative is as follows: $g_{a b}^{\prime} \equiv \partial g_{a b} / \partial r$ and $r^{\prime} \equiv d r / d x_{1}$. Generically, it is very difficult to draw any conclusion on the determinant of matrix $M$. However, it is easy to show that the quantity $A$ is positive. This follows by considering the solution that follows, in fact as constructed in [12], $r^{\prime}=\sqrt{g_{x x}^{d}(r)-g_{x x}^{d-1}\left(r_{\star}\right) g_{x x}(r)} / g_{x x}^{(d-1) / 2}\left(r_{\star}\right) \sqrt{g_{r r}(r)}$, in which case

$$
A=\frac{g_{r r}(r) g_{x x}^{3(d-1) / 2}\left(r_{\star}\right)}{g_{x x}^{d}(r)}>0,
$$


and the expressions for $\operatorname{det}(M)$ are very cumbersome to write down explicitly. The quantity, $r_{\star}$, is determined by requiring that $r^{\prime}$ vanishes there.

Note that $\left(1 / L^{d-2}\right) \delta^{2}(\operatorname{Area}(\Sigma)) / \delta r^{\prime} \delta r^{\prime}=2 A$. In order to check the weak minimality condition on the area functional, we need to look at the condition $A>0$, which is obeyed automatically. Now moving on to determine the sign of the determinant of the matrix $M$, generically, it is very difficult to draw any conclusion. Nevertheless, we will check it on caseby-case basis.

AdS. To begin with, let us consider the AdS spacetime with radius $R$ and the boundary is at $r=0$, in which case

$$
\begin{gathered}
A=r^{2(d-1)} R^{d-1} r_{\star}^{-3(d-1)}, \\
B=-\frac{2(d-1)}{r} R^{d-1} \sqrt{r^{2-2 d}-r_{\star}^{2-2 d}}, \\
C=\frac{d(d-1)}{r^{2 d}}\left(R r_{\star}\right)^{d-1}
\end{gathered}
$$

where we have considered $g_{x x}=R^{2} / r^{2}=g_{r r}$. The quantity

$$
\begin{aligned}
& \frac{4 A C-B^{2}}{4} \\
& \quad=(d-1)^{2} \frac{R^{2(d-1)}}{r^{2 d}}\left[\frac{(2 d-1)}{(d-1)}\left(\frac{r}{r_{\star}}\right)^{2(d-1)}-1\right] .
\end{aligned}
$$

We know that the surface under study starts from the boundary $r=0$ and goes all the way to $r=r_{\star}$ but does not go past $r=r_{\star}$, which means the above quantity is positive only close to $r_{\star}$, whereas close to UV, it becomes negative. This result suggests that the weak minimum is not a sufficient condition.

$H S V$. For hyperscale violating (HSV) solution in the convention of [26] with $g_{x x}=R^{2} / r^{2-2 \gamma}=g_{r r}$ where $\gamma$ is a constant, the positivity of $A$ is easy to observe whereas the $\operatorname{det}(M)$ is

$$
\begin{aligned}
\frac{4 A C-B^{2}}{4}= & -(d-1)^{2}(\gamma-1)^{2} \frac{R^{2(d-1)}}{r^{2 d-2 \gamma(d-1)}} \\
& \cdot\left[\frac{(2 d-1-2 \gamma(d-1))}{(\gamma-1)(d-1)}\left(\frac{r}{r_{\star}}\right)^{2(d-1)(1-\gamma)}+1\right] .
\end{aligned}
$$

It is easy to see again that, close to UV, the $\operatorname{det}(M)$ becomes negative and becomes positive close to $r_{\star}$ for both positive and negative $\gamma$ 's.

Black Hole. Let us consider a black hole; for simplicity, we assume it asymptotes to AdS spacetime with the boundary to be at $r=0$. In this coordinate system the horizon is located at $r=r_{h}>0$. Moreover, $g_{x x}(r)$ is positive for all values of $r$ and it takes the following form:

$$
g_{r r}(r)=\left\{\begin{array}{lll}
+\mathrm{ve} & \text { for } r<r_{h} & \text { (Outside the horizon) } \\
-\mathrm{ve} & \text { for } r>r_{h} & \text { (Inside the horizon) } .
\end{array}\right.
$$

It follows from (15) that as the hypersurface goes inside the black hole, the quantity, $A$, becomes negative, whereas outside the horizon, it stays positive. So, we see that if the hypersurface stays outside the horizon, as suggested in $[16,17]$, then it follows naturally that there exists a (weak) minimality condition on the area functional.

In order to check the sign of the determinant of the matrix $M$, let us take the following choice of the metric components:

$$
\begin{gathered}
g_{x x}=\frac{R^{2}}{r^{2}}, \quad g_{r r}=\frac{R^{2}}{\left(r^{2} f(r)\right)}, \\
f(r)=1-\left(\frac{r}{r_{h}}\right)^{d} .
\end{gathered}
$$

In this case, we get

$$
\begin{aligned}
A= & R \frac{r^{d-1}}{f(r)} r_{\star}^{-3(d-1)}, \\
B= & R^{d-1} r_{h}^{d / 2} \frac{\sqrt{r^{2(1-d)}-r_{\star}^{2(1-d)}}}{r^{3} r_{\star}^{2 d}\left(r_{h}^{d}-r^{d}\right)^{3 / 2}} \\
& \cdot\left(d\left[r^{3 d} r_{\star}^{2}+r_{\star}^{2 d}\left(3 r^{d+2}-2 r^{2} r_{h}^{d}\right)\right]-2 r^{2} r_{\star}^{2 d}\left(r^{d}-r_{h}^{d}\right)\right), \\
C= & d R^{d-1} r^{2(d-2)} r_{\star}^{d-1} \\
& \cdot\left((9 d-6) r^{2(2-d)}-10(d-1) r^{4-3 d} r_{h}^{d}\right. \\
& +4(d-1) r^{4(1-d)} r_{h}^{2 d} \\
& \quad d r^{2 d} r_{\star}^{4(1-d)}-2(2 d-1) r^{2} r_{\star}^{2(1-d)} \\
& \left.+2(d-1) r^{2-d} r_{h}^{d} r_{\star}^{2(1-d)}\right),
\end{aligned}
$$

where $r_{\star}$ is the turning point of the solution, which is the maximum reach of the hypersurface in the bulk.

Let us rescale $r=u r_{\star}$ and $r_{h}=n r_{\star}$, so that $u$ and $n$ are dimensionless. For simplicity, we take $d=4$, in which case

$$
\begin{aligned}
4 A C-B^{2}= & -4 n^{4} R^{6} \\
& \cdot\left(3 n^{8}\left(7 u^{6}-3\right)-6 u^{4} n^{4}\left(u^{12}-5+8 u^{6}\right)\right. \\
& \left.+u^{8}\left(2 u^{12}-25+35 u^{6}\right)\right) \\
& \cdot\left(r_{\star}^{8} u^{8}\left(u^{4}-n^{4}\right)^{3}\right)^{-1} .
\end{aligned}
$$

Generically, $\left(r_{\star}^{8} / R^{6}\right)\left(4 A C-B^{2}\right)$ is a function of two variables $n$ and $u$. It is very easy to see that, close to UV, that is, for very small values of $u$, the function $\left(r_{\star}^{8} / R^{6}\right)\left(4 A C-B^{2}\right)$ becomes negative. It means that close to the boundary the determinant of matrix $M$ is not positive. So the weak minimality condition is not a sufficient condition.

Let us recall that $r=(u / n) r_{h}$. It means that when $u>n$ we are inside the horizon and when $u<n$ we are outside 
the horizon. If the turning point $r_{\star}$ is inside the horizon, then $r=r_{\star}>r_{h}$. This means that $n<1$. Similarly, for $r_{\star}$ outside the horizon, $r=r_{\star}<r_{h}$, which means that $n>1$. In summary,

$$
\begin{array}{ll}
u<n, \quad n>1 \quad \text { (Outside the horizon) } \\
u>n, \quad n<1 \quad \text { (Inside the horizon). }
\end{array}
$$

For simplicity, we will restrict $n$ to stay from $1<n \leq 2$ for outside the horizon which means $0 \leq u<1$, whereas, for inside the horizon, we will take $0 \leq n<1$ which means $1<$ $u \leq 2$.

The quantity $\left(r_{\star}^{8} / R^{6}\right)\left(4 A C-B^{2}\right)$ is plotted inside the horizon for AdS black hole in $4+1$-dimensional spacetime in Figure 1. It is easy to notice that this quantity is always negative inside the horizon of the AdS black hole.

Both the quantities, $A$, and the determinant of the matrix, $M$, become negative. This simply means that there does not exist any hypersurface inside the horizon that minimizes the area functional. Recall that, according to Ryu-Takayanagi conjecture, we need to find the area of the hypersurface that minimizes the area functional in the computation of the entanglement entropy. So, we can interpret the absence of the minimal area hypersurface inside the horizon as the nonpenetration of such hypersurface into the horizon. This conclusion is in perfect agreement with that reached in $[16$, 17].

Outside the Horizon. Let us look at the behavior of the quantities $A$ and $\operatorname{det}(M)$ outside the horizon. It is easy to see that the quantity $A$ is always positive outside the horizon, which follows simply from (15). The information about the other quantity, namely, the determinant of the matrix $M$, can be obtained numerically, which is plotted in Figure 2 .

It is clear from Figure 2 that the determinant of the matrix $M$ becomes negative close to UV, which suggests that the Jacobi condition for the sufficient nature of the weak minimality condition does not hold.

Confining Solution. Let us study the weak minimality condition on the area functional in the case for which the background solution shows confining behavior. To generate such a confining background, the easiest method is to start with the uncharged black hole solution and perform a double Wick rotation. In the end, the solution that asymptotes to $\mathrm{AdS}_{d+1}$ with unit AdS radius reads as

$$
\begin{gathered}
d s_{d+1}^{2}=\frac{1}{r^{2}}\left(-d t^{2}+f(r) d x_{1}^{2}+d x_{2}^{2}+\cdots+d x_{d-1}^{2}\right) \\
+\frac{d r^{2}}{r^{2} f(r)}, \\
f(r)=1-\frac{r^{d}}{r_{0}^{d}} .
\end{gathered}
$$

The coordinate $x_{1}$ is now periodic with periodicity $2 \pi \beta$, whose explicit form is not important for us. The IR is at $r=r_{0}$ and the UV is at $r=0$. We can proceed further by studying two cases, depending on the fields that we are exciting.

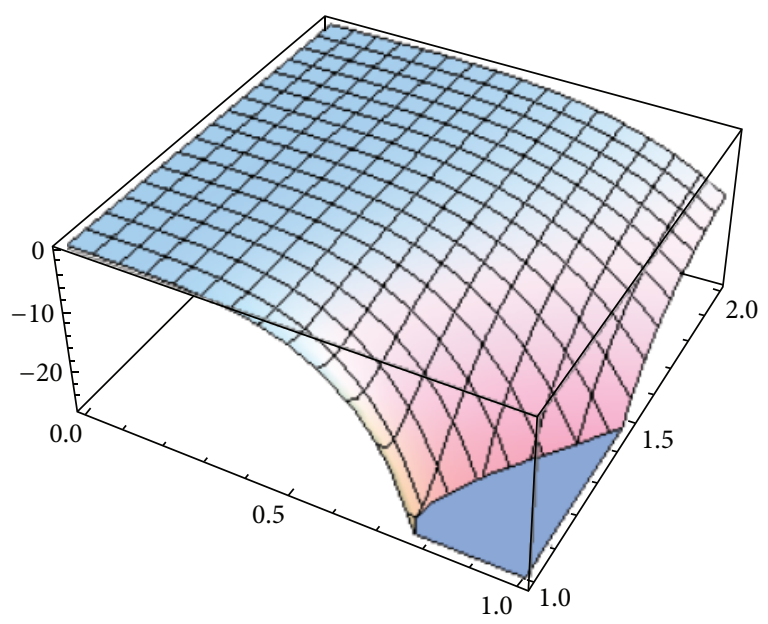

FIgURE 1: $\left(r_{\star}^{8} / R^{6}\right)\left(4 A C-B^{2}\right)$ is plotted for $\mathrm{AdS}_{5}$ black hole inside the horizon for which $0 \leq n<1$ and $1<u \leq 2$.

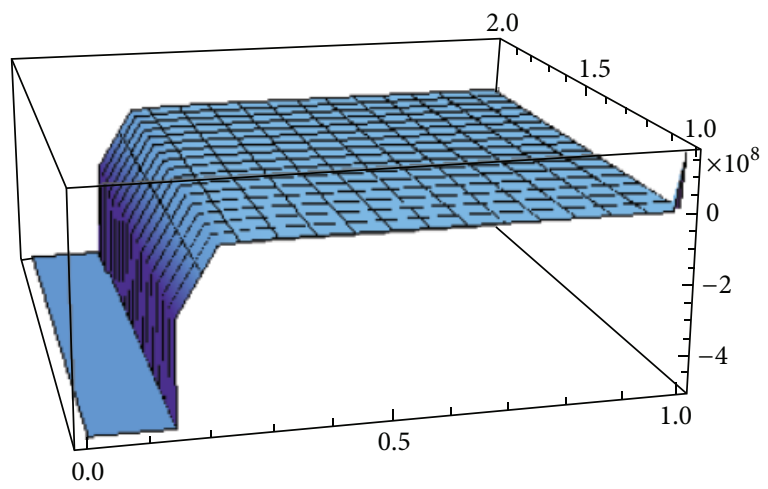

FIGURE 2: The figure is plotted for $\left(r_{\star}^{8} / R^{6}\right)\left(4 A C-B^{2}\right) r \mathrm{AdS}_{5}$ black hole outside the horizon for which $0 \leq u<1$ and $1<n \leq 2$.

Case 1. The induced metric on the codimension twohypersurface takes the following form:

$$
\begin{gathered}
d s_{d-1}^{2}=\frac{1}{r^{2}}\left(f(r) d x_{1}^{2}+d x_{3}^{2}+\cdots+d x_{d-1}^{2}\right) \\
+\left(1+\frac{r^{\prime 2}}{f(r)}\right) \frac{d x_{2}^{2}}{r^{2}}, \\
r^{\prime}=\frac{d r}{d x_{2}} .
\end{gathered}
$$

In this case, the area of the induced geometry for the strip times a shrinking circle type entangling region, $0 \leq x_{1} \leq$ $2 \pi \beta, 0 \leq x_{2} \leq \ell,-L / 2 \leq\left(x_{3}, \ldots, x_{d-1}\right) \leq L / 2$, becomes

$$
A=\int d x_{1} \cdots d x_{d-1} \frac{\sqrt{f(r)+r^{\prime 2}}}{r^{d-1}} .
$$
form:

The solution to the equation of motion takes the following

$$
\frac{d r}{d x_{2}}=\frac{\sqrt{f\left(f-c_{0}^{2} r^{2(d-1)}\right)}}{c_{0} r^{d-1}},
$$


where the constant of integration $c_{0}$ is determined as follows: $\left(d r / d x_{2}\right)_{r_{\star}} \rightarrow 0$. This means that $c_{0}=\sqrt{f\left(r_{\star}\right)} / r_{\star}^{d-1}$. The second variation of the area functional can be written as follows:

$$
\delta^{2} \text { Area }=\int \prod_{i} d x_{i}\left(A \delta r^{\prime} \delta r^{\prime}+\widetilde{A} \delta r \delta r\right),
$$

where we have dropped a boundary term using the boundary conditions $\delta r(0)=0$ and $\delta r(\ell)=0$. The quantities are

$$
\begin{gathered}
A=\frac{1}{r^{d-1}\left(f(r)+r^{\prime 2}\right)^{3 / 2}}, \\
\widetilde{A}=\frac{d(d-1)}{r^{d+1}} \sqrt{f(r)+r^{\prime 2}} \\
-\frac{(d-1)}{r^{d} \sqrt{f(r)+r^{\prime 2}}} \frac{d f}{d r}+\frac{d}{d x_{2}}\left(\frac{(d-1) r^{\prime}}{r^{d} \sqrt{f(r)+r^{\prime 2}}}\right) \\
-\frac{1}{4 r^{d-1}\left(f+r^{\prime 2}\right)^{3 / 2}}\left(\frac{d f}{d r}\right)^{2} \\
+\frac{d}{d x_{2}}\left(\frac{r^{\prime}(d f / d r)}{2 r^{d-1}\left(f(r)+r^{\prime 2}\right)^{3 / 2}}\right) \\
+\frac{1}{2 r^{d-1} \sqrt{f(r)+r^{\prime 2}}} \frac{d^{2}}{d r^{2}} f .
\end{gathered}
$$

Once again we can introduce the function $W$ as is done in the introduction and finally we are interested in the quantity $A$. Using the solution for $r^{\prime}$ results in

$$
A=\frac{r^{2(d-1)} c_{0}^{3}}{(f(r))^{3}}>0 .
$$

It is easy to see the positivity of $A$, because the radial coordinate stays from $0 \leq r \leq r_{0}$. Hence, the weak minimality of the area functional for this case is checked.

Case 2. In this case, we consider that the embedding field as studied in [27], that is, the field, $r$, which is excited is a function of the compact coordinate $x_{1}$. In the $\left(r, x_{1}\right)$ plane, it will be a cigar. In this case, the induced geometry reads as

$$
\begin{gathered}
d s_{d-1}^{2}=\frac{1}{r^{2}}\left(d x_{2}^{2}+d x_{3}^{2}+\cdots+d x_{d-1}^{2}\right) \\
+\left(\frac{f^{2}+r^{\prime 2}}{r^{2} f}\right)+d x_{1}^{2}, \\
r^{\prime}=\frac{d r}{d x_{1}} .
\end{gathered}
$$

The area functional reads as

$$
\text { Area }=\int d x_{1} \cdots d x_{d-1}\left(\frac{\sqrt{f^{2}+r^{\prime 2}}}{r^{d-1} \sqrt{f}}\right) .
$$

The equation of motion that follows gives the following solution:

$$
r^{\prime}=\frac{f(r) \sqrt{f(r)-c_{0}^{2} r^{2(d-1)}}}{c_{0} r^{d-1}},
$$

where the constant of integration, $c_{0}$, is found by demanding that the quantity, $r^{\prime}$, vanishes in the limit $r \rightarrow r_{\star}$. It sets $c_{0}=\sqrt{f\left(r_{\star}\right)} / r_{\star}^{d-1}$. Finding the second variational of the area functional using the boundary conditions $\delta r(0)=0$ and $\delta r(2 \pi \beta)=0$ gives

$$
\delta^{2} \text { Area }=\int \prod_{i} d x_{i}\left(A \delta r^{\prime} \delta r^{\prime}+\widetilde{A} \delta r \delta r\right) .
$$

For our purpose, the precise form of the quantity $\widetilde{A}$ is not important as we are interested in finding only the form of $A$ and its sign. In the present case, it reads as

$$
A=\frac{1}{r^{d-1}\left(f(r)+r^{\prime 2} / f(r)\right)^{3 / 2}} .
$$

Using the solution as written above, it is easy to conclude that

$$
A=\frac{r^{2(d-1)} c_{0}^{3}}{(f(r))^{3}}>0
$$

It is interesting to note that the quantity, $A$, in both cases, $r\left(x_{1}\right)$ and $r\left(x_{2}\right)$, gives a minimum to the area functional.

As an aside, the existence of two valid configurations means that there can be a phase transition induced quantum mechanically depending on the energy of these two configurations, which is studied in detail in [27]. But for our purposes we see that both are becoming minima to the area functional, which we set out to find.

2.1. Sphere. Let us consider another example, where the entangling region, $\Sigma$, is of the sphere type. In this context, we assume that the bulk geometry is

$$
\begin{aligned}
d s_{d+1}^{2}= & -g_{t t}(r) d t^{2}+g_{x x}(r)\left(d x_{1}^{2}+\cdots+d x_{d-1}^{2}\right) \\
& +g_{r r}(r) d r^{2} \\
= & -g_{t t} d t^{2}+g_{x x}\left(d \rho^{2}+\rho^{2} d \Omega_{d-2}^{2}\right)+g_{r r} d r^{2}
\end{aligned}
$$

Using the Ryu-Takayanagi prescription, the geometry of the codimension two-hypersurface takes the following form:

$$
d s_{d-1}^{2}=\left(g_{x x}+g_{r r} r^{\prime 2}\right) d \rho^{2}+g_{x x} \rho^{2} d \Omega_{d-2}^{2},
$$

where $r^{\prime}=d r / d \rho$. The area functional reads as

$$
\begin{aligned}
\operatorname{Area}(\Sigma) & =\omega_{d-2} \int d \rho \rho^{d-2} g_{x x}^{(d-2) / 2} \sqrt{g_{x x}+g_{r r} r^{\prime 2}} \\
& =\omega_{d-2} \int d r \rho^{d-2} g_{x x}^{(d-2) / 2} \sqrt{g_{x x} \rho^{\prime 2}+g_{r r}},
\end{aligned}
$$


where $\omega_{d-2}$ is the volume form associated with the unit $d-2$ dimensional sphere, $S^{d-2}$. The equation of motion that follows takes the following form:

$$
\begin{gathered}
\partial_{r}\left(\frac{\rho^{d-2} g_{x x}^{d / 2} \rho^{\prime}}{\sqrt{g_{x x} \rho^{\prime 2}+g_{r r}}}\right) \\
\quad-(d-2) \rho^{d-3} g_{x x}^{(d-2) / 2} \sqrt{g_{x x} \rho^{\prime 2}+g_{r r}}=0,
\end{gathered}
$$

where $\rho^{\prime}=d \rho / d r$. Upon considering the background geometry as AdS spacetime with radius $R, g_{x x}=g_{r r}=$ $R^{2} / r^{2}$, the solution that follows takes the following form: $\rho=$ $\sqrt{c^{2}-r^{2}}$, where $c$ is a constant of integration.

Let us find the second variation of the area functional as written in (39) for AdS spacetime:

$$
\delta^{2} \text { Area }(\Sigma)=\omega_{d-2} R^{d-1} \int d \rho\left[A\left(\delta r^{\prime}\right)^{2}+B \delta r \delta r^{\prime}+C(\delta r)^{2}\right],
$$

where

$$
\begin{aligned}
& A=\frac{\rho^{d-2}}{r^{d-1}\left(1+r^{\prime 2}\right)^{3 / 2}}, \\
& B=-2(d-1) \frac{r^{\prime} \rho^{d-2}}{r^{d} \sqrt{1+r^{\prime 2}}}, \\
& C=d(d-1) \frac{\rho^{d-2} \sqrt{1+r^{\prime 2}}}{r^{d+1}} .
\end{aligned}
$$

In getting the above-mentioned second variation of the area functional, we have used the equation of motion obeyed by $r=r(\rho)$. Computing the following quantity:

$$
\begin{aligned}
\frac{\delta^{2}(\text { Area }(\Sigma))}{\delta r^{\prime} \delta r^{\prime}} & =2 \frac{\omega_{d-2} g_{x x}^{d / 2} g_{r r} \rho^{d-2}}{\left(g_{x x}+r^{\prime 2} g_{r r}\right)^{3 / 2}} \\
& =2 \frac{\omega_{d-2} R^{d-1} \rho^{d-2}}{c^{3}\left(c^{2}-\rho^{2}\right)^{(d-4) / 2}} \\
& =2 \frac{\omega_{d-2} R^{d-1} \rho^{d-2}}{c^{3} r^{d-4}}>0 .
\end{aligned}
$$

In getting the second equality, we have used the geometry of AdS spacetime. Note that both $r$ and $\rho$ are real and positive; hence the above quantity, $A$, is positive.

Let us determine the sign associated with the determinant of matrix $M$. In this case,

$$
\begin{aligned}
4 A C-B^{2} & =-4(d-1) \rho^{2(d-2)}\left(\frac{(d-1) r^{\prime 2}-d}{r^{2 d}\left(1+r^{\prime 2}\right)}\right) \\
& =4(d-1) \rho^{2(d-2)}\left(\frac{\rho^{2}+d\left(r^{2}-\rho^{2}\right)}{c^{2} r^{d / 2}}\right) .
\end{aligned}
$$

Using the following hypersurface: $r=\sqrt{c^{2}-\rho^{2}}$ close to UV, we find that the quantity, $4 A C-B^{2}$, close to UV becomes negative and large, which means that the determinant of the matrix $M$ is negative. Note that the constant $c$ can be identified with the size of the sphere, $R$.

\section{With Higher Derivative}

In the presence of the higher derivative term in the entanglement entropy functional, it is not a priori clear that the entanglement entropy functional will be a minimum, automatically. Moreover, we cannot apply (5) in the determination of the Legendre test. However, it is suggested in $[18,20]$ that for a very specific type of entanglement entropy functional one can get a minimal entanglement entropy functional. In the present case, we will determine the consequence of the imposition of the minimal nature of the entanglement entropy functional for the AdS spacetime only, which depends crucially on the value of the coupling, $\lambda_{1}$, as defined later. The precise form of the entanglement entropy functional with the higher derivative term can be considered as described by the Jacobson-Myers functional [28]. In fact, for our purpose, we will consider the structure as studied in [21-23]

$$
4 G_{N} S_{E E}=\int d^{d-1} \sigma \sqrt{\operatorname{det}\left(g_{a b}\right)}\left[1+\lambda_{1} R(g)\right]
$$

where $\lambda_{1}$ is the coupling constant and defined (This follows by comparing with the action used in [23].) as $\lambda_{1} \equiv 2 \lambda_{a} R_{A}^{2} /(d-$ $2)(d-3)$. Let us evaluate the entanglement entropy for the strip type entangling region as discussed earlier. Using the structure of the induced metric $g_{a b}$ as written down in (11) gives [12]

$$
\begin{gathered}
2 G_{N} S_{E E} \\
=L^{d-2} \int d r \frac{g_{x x}^{(d-6) / 2}}{4\left[g_{r r}+g_{x x} x_{1}^{\prime 2}\right]^{3 / 2}} \\
\cdot\left[4 g_{x x}^{2}\left(g_{r r}+g_{x x} x_{1}^{\prime 2}\right)^{2}+\lambda_{1}(d-2)\right. \\
\cdot\left(2 g_{x x} g_{x x}^{\prime} g_{r r}^{\prime}-(d-7) x_{1}^{\prime 2} g_{x x} g_{x x}^{\prime 2}\right. \\
+4 x_{1}^{\prime} x_{1}^{\prime \prime} g_{x x}^{2} g_{x x}^{\prime}-4 x_{1}^{\prime 2} g_{x x}^{2} g_{x x}^{\prime \prime} \\
\left.\left.-4 g_{x x} g_{r r} g_{x x}^{\prime \prime}-(d-5) g_{r r} g_{x x}^{\prime 2}\right)\right]
\end{gathered}
$$

where $x_{1}^{\prime}=d x_{1} / d r$. This for the AdS spacetime (Such solutions and the associated phase transitions with the black hole solutions are studied in great detail in, for example, 
[29-34].) with the boundary at $r=0$ and with the AdS radius $R_{0}$ becomes

$$
\begin{aligned}
& 2 G_{N} S_{E E} \\
& =L^{d-2} R_{0}^{d-1} \\
& \cdot \int d x_{1}\left[\frac{\sqrt{1+r^{\prime 2}}}{r^{d-1}}-\frac{(d-2) \lambda_{1}}{R_{0}^{2}}\right. \\
& =L^{d-2} R_{0}^{d-1} \cdot\left(\frac{(d-1) r^{\prime 2}}{\left.\left.r^{d-1} \sqrt{1+r^{\prime 2}}-\frac{2 r^{\prime \prime}}{r^{d-2}\left(1+r^{\prime 2}\right)^{3 / 2}}\right)\right]}\right. \\
& \cdot \int d r\left[\frac{\sqrt{1+x_{1}^{\prime 2}}-\frac{(d-2) \lambda_{1}}{R_{0}^{2}}}{\left.r^{d-1}-\left(\frac{(d-1)\left(1+x_{1}^{\prime 2}\right)+2 r x_{1}^{\prime} x_{1}^{\prime \prime}}{r^{d-1}\left(1+x_{1}^{\prime 2}\right)^{3 / 2}}\right)\right]}\right.
\end{aligned}
$$
form:

The equation of motion that follows takes the following

$$
\frac{d}{d r}\left[\frac{x_{1}^{\prime}}{r^{d-1} \sqrt{1+x_{1}^{\prime 2}}}-\frac{(d-2)(d-3) \lambda_{1} x_{1}^{\prime}}{R_{0}^{2} r^{d-1}\left(1+x_{1}^{\prime 2}\right)^{3 / 2}}\right]=0 .
$$

The second variation of the entanglement entropy functional can be expressed as

$$
\begin{aligned}
& 2 G_{N} \delta^{2} S_{E E} \\
& =L^{d-2} R_{0}^{d-1} \\
& \quad \cdot \int d x_{1}\left(A \delta r^{\prime} \delta r^{\prime}+B \delta r \delta r^{\prime}+C \delta r \delta r+D \delta r \delta r^{\prime \prime}+E \delta r^{\prime} \delta r^{\prime \prime}\right) \\
& =L^{d-2} R_{0}^{d-1} \int d x_{1} V^{T} \cdot M \cdot V,
\end{aligned}
$$

where the column vector $V$ and the matrix $M$ are

$$
\begin{gathered}
V=\left(\begin{array}{c}
\delta r^{\prime} \\
\delta r \\
\delta r^{\prime \prime}
\end{array}\right), \quad M=\left(\begin{array}{ccc}
A & \frac{B}{2} & \frac{E}{2} \\
\frac{B}{2} & C & \frac{D}{2} \\
\frac{E}{2} & \frac{D}{2} & 0
\end{array}\right), \\
\operatorname{det}(M)=\frac{E(B D-C E)-A D^{2}}{4} .
\end{gathered}
$$

The various expressions are

$$
\begin{aligned}
A= & \frac{1}{r^{d-1}\left(1+r^{\prime 2}\right)^{3 / 2}}-\frac{(d-2) \lambda_{1}}{R_{0}^{2}} \\
& \cdot\left(\frac{2(d-1)-3(d-1) r^{\prime 2}}{r^{d-1}\left(1+r^{\prime 2}\right)^{3 / 2}}\right. \\
& +\frac{3(d-1) r^{\prime 4}}{r^{d-1}\left(1+r^{\prime 2}\right)^{5 / 2}}+\frac{6 r^{\prime \prime}}{r^{d-2}\left(1+r^{\prime 2}\right)^{5 / 2}} \\
& \left.-\frac{30 r^{\prime 2} r^{\prime \prime}}{r^{d-2}\left(1+r^{\prime 2}\right)^{7 / 2}}\right), \\
B= & -\frac{2(d-1) r^{\prime}}{r^{d} \sqrt{1+r^{\prime 2}}}-\frac{(d-2) \lambda_{1}}{R_{0}^{2}} \\
& \cdot\left(\begin{array}{l}
-\frac{4(d-1)^{2} r^{\prime}}{r^{d} \sqrt{1+r^{\prime 2}}}+\frac{2(d-1)^{2} r^{\prime 3}}{r^{d}\left(1+r^{\prime 2}\right)^{3 / 2}} \\
\left.-\frac{12(d-2) r^{\prime} r^{\prime \prime}}{r^{d-1}\left(1+r^{\prime 2}\right)^{5 / 2}}\right)
\end{array}\right.
\end{aligned}
$$$$
C=\frac{d(d-1) \sqrt{1+r^{\prime 2}}}{r^{d+1}}
$$$$
\begin{gathered}
-\frac{(d-2) \lambda_{1}}{R_{0}^{2}}\left(\frac{d(d-1)^{2} r^{\prime 2}}{r^{d+1} \sqrt{1+r^{\prime 2}}}-\frac{2(d-1)(d-2) r^{\prime \prime}}{r^{d}\left(1+r^{\prime 2}\right)^{3 / 2}}\right), \\
D=-\frac{(d-2) \lambda_{1}}{R_{0}^{2}} \frac{4(d-2)}{r^{d-1}\left(1+r^{\prime 2}\right)^{3 / 2}} \\
E=-\frac{(d-2) \lambda_{1}}{R_{0}^{2}} \frac{12 r^{\prime}}{r^{d-2}\left(1+r^{\prime 2}\right)^{5 / 2}} .
\end{gathered}
$$

Now, we can demand the Legendre condition as stated earlier and, at the end, we are interested in determining under what condition the quantity $A$ is positive. Using the real valued solution that follows from (48) to the leading order in the coupling $\lambda_{1}$ gives

$$
A=r^{2(d-1)} r_{\star}^{-3(d-1)}\left(1-2(d-1)(d-2) \frac{\lambda_{1}}{R_{A}^{2}}\right)+\mathcal{O}\left(\lambda_{1}\right)^{2},
$$

where we have used the relationship between the sizes of the AdS spacetime, $R_{0}$ and $R_{A}$. The size $R_{A}$ is defined in the infinite 't Hooft coupling limit and is related to $R_{0}=$ $R_{A} / \sqrt{f_{\infty}}$, where $f_{\infty}$ obeys the following relation: $1-f_{\infty}+$ $\lambda_{a} f_{\infty}^{2}=0$; see, for example, [23, 29]. Demanding that the quantity $A$ is positive gives the following restriction on the coupling:

$$
\lambda_{1}<\frac{R_{A}^{2}}{2(d-1)(d-2)} .
$$


Using the couplings used in [23], we can rewrite (where $\lambda_{a}$ here is the same as $\lambda$ in [23].) the coupling $\lambda_{1}=$ $2 \lambda_{a} R_{A}^{2} /(d-2)(d-3)$, in which case

$$
\lambda_{a}<\frac{d-3}{4(d-1)}
$$

The inclusion of the finite ' $t$ Hooft coupling correction to the entanglement entropy functional does not automatically make the entanglement entropy functional a minimum (it is suggested in [18] that when the extra piece other than the area of the codimension two-surface term in the entanglement entropy functional has the form of $f(R)$, where $R$ is the induced scalar curvature of the codimension two-surface, then one expects to have a minimum in the entanglement entropy functional).

Demanding the minimal condition on the entanglement entropy functional puts a restriction on the coupling as written in (53) and (54). Hence, we can interpret that the minimality condition essentially says that the coupling has an upper bound which is positive. Moreover, the Jacobi test in the present case does not give anything interesting to leading linear order in $\lambda_{1}$, as the terms $D E, E^{2}$, and $D^{2}$ in the $\operatorname{det}(M)$ are quadratic order in $\lambda_{1}$.

\section{Discussion}

It is suggested in [20] that the strong subadditivity property (The strong subadditivity property, $S(A)+S(B) \geq S(A \cup$ $B)+S(A \cap B)$, is proven in the holographic case but without the higher derivative term in [35]. It is certainly interesting to ask whether the entanglement entropy functional as suggested, generically, in $[18,19]$ do automatically respect the strong subadditivity property and the hypersurface under study becomes a minimal surface. Moreover, we need to find the precise connection between the strong subadditivity and the minimal hypersurface.) should be obeyed by the entanglement entropy functional (45), and the integration is done over a hypersurface which minimizes the entanglement entropy functional. We noticed that such minimality of the entanglement entropy functional does not happen for all values of the couplings, $\lambda_{a}$; however, it does happen only when we put a serious restriction on the coupling $\lambda_{a}$ as in (54). Hence, the imposition of the minimization condition on the entanglement entropy functional with the higher derivative term as suggested in [20] puts a restriction on the coupling $\lambda_{a}$.

In $4+1$-dimensional AdS spacetime, it is suggested in [36] using the positivity of the energy fluxes and the causality that the Gauss-Bonnet (GB) coupling stays in a small window and can become a small negative number to a small positive number, which in our notation becomes $-7 / 36 \leq \lambda_{a} \leq 9 / 100$. From the study of the minimality of the entanglement entropy functional, we find, for $d=4$, that the coupling should have an upper bound; that is, $\lambda_{a}<1 / 12$. It is not known how to fix the lower bound.
Generalizing it to arbitrary $d+1$-dimensional spacetime, it is found in $[24,25,37-39]$ that the coupling, in our notation, should stay in the following range:

$$
-\frac{(d-2)(3 d+2)}{4(d+2)^{2}} \leq \lambda_{a} \leq \frac{(d-2)(d-3)\left(d^{2}-d+6\right)}{4\left(d^{2}-3 d+6\right)^{2}} .
$$

It is interesting to note that, in the large $d$ limit, $d \rightarrow \infty$, both (54) and (55) give the same upper bound, namely, $1 / 4$.

The disagreement on the range of the GB coupling suggests that the hypersurface under study does not necessarily minimize the entanglement entropy. (Let us note that the constraint on the coupling $\lambda_{a}$ follows (from (54)) by doing an analysis only to leading order in the coupling.) Hence, it remains an open question to know the precise nature of the hypersurface with higher derivative term in the entanglement entropy.

\section{Conclusion}

The Ryu-Takayanagi (RT) conjecture gives an interesting proposal to calculate the entanglement entropy using a gravitational description. For a fixed time slice, the RT conjecture states that the entanglement entropy functional is described by the area of a codimension two-hypersurface. Moreover, the codimension two-hypersurface should be determined in such a way that it minimizes the entanglement entropy functional. In this paper, we have studied the consequences of the minimality condition on the entanglement entropy functional, especially by performing the Legendre test and the Jacobi test. We have checked, for the strip type entangling region, by studying various examples like thermal AdS solution, confining solution, hyperscale violating solution, and the black holes in the AdS spacetime that it obeys necessarily the (weak) minimality condition but not the sufficient condition.

For our purpose, the outside of the black hole is described by the radial coordinate that stays from the boundary $r=0$ to the horizon, $r=r_{h}$, whereas the inside is described by $r>r_{h}$. Let us recall from the second variation of the area functional equation (13) that it is the sign of the quantity, $A$, that determines whether the area functional is a minimum or a maximum. It is easy to notice, using the property of $g_{r r}$ as mentioned in Section 2 and from (15), that as long as we stay outside of the horizon, it gives a minimum. Once we are inside the horizon, it gives a maximum. So, we may interpret that it is the horizon that acts as a surface which separates the minimum area functional from the maximum. Hence, we can say that it is the RT conjecture that leads naturally to the following conclusion: we better stay outside of the horizon if we want a minimum area. This finally allows us to conclude that the minimality of the area functional does not allow the codimension two-hypersurface to enter into the black hole horizon. The same conclusion is reached (In this case there does not exist any real valued solution of the embedding field, $X^{M}$, inside the horizon.) in [17] and more generally in [16].

In a recent study in [16], it is argued that regions with negative extrinsic curvature cannot be accessed by 
any hypersurfaces irrespective of whether it is of spacelike, timelike, or null type. Let us recall that the imposition of the (weak) minimality condition gives us (5), which is negative inside the horizon. A priori, it is not clear whether there exists any relationship (One way to look at is as follows: the number of free indices that appear in (5) is four whereas, in the definition of the extrinsic curvature as in [16], it can be of maximum three, for a hypersurface of codimension bigger than unity.) between the extrinsic curvature studied in [16] and (5). However, we do expect that there should exist some kind of relation between these quantities because of the similarity in their behavior. In particular, for the black hole geometry, the quantity, $A$, as written in (15) shows

$$
A^{-1}=\left\{\begin{array}{lll}
+\mathrm{ve} & \text { for } r<r_{h} & \text { (Outside the horizon) } \\
0 & \text { for } r=r_{h} & \text { (On the horizon) } \\
-\mathrm{ve} & \text { for } r>r_{h} & \text { (Inside the horizon) }
\end{array}\right.
$$

The extrinsic curvature shows precisely the similar type of behavior as reported in [16]. The connections between these two quantities are worth studying, which we leave for future studies.

Moving onto the calculation of the entanglement entropy with higher derivative term, it is argued in [20] that the hypersurface should be minimal when the entanglement entropy functional is described by (45). Upon applying such a minimality condition, it imposes an important restriction on the Gauss-Bonnet coupling, $\lambda_{1}$. This is given in (53) and (54), which essentially gives an upper bound on the coupling. The bound so obtained does not match precisely that derived in $[24,25]$ using the positivity of the energy fluxes and the causality constraint. Hence, it is highly plausible that theories with higher derivative term in the entanglement entropy functional do not have hypersurfaces that are either minimal or maximal in nature. So, the question about its nature remains to be seen in future studies.

\section{Conflict of Interests}

The authors declare that there is no conflict of interests regarding the publication of this paper.

\section{Acknowledgments}

The authors are pleased to thank the Almighty for giving them enough energy to do this work. Shesansu Sekhar Pal would like to thank the computer center, IoP, Bhubaneswar.

\section{References}

[1] S. Ryu and T. Takayanagi, "Holographic derivation of entanglement entropy from the anti-de Sitter space/conformal field theory correspondence," Physical Review Letters, vol. 96, no. 18, Article ID 181602, 2006.

[2] S. Ryu and T. Takayanagi, "Aspects of holographic entanglement entropy," Journal of High Energy Physics, vol. 2006, no. 8, article 45, 2006.
[3] E. Bianchi and R. C. Myers, "On the architecture of spacetime geometry," Classical and Quantum Gravity, vol. 31, no. 21, Article ID 214002, 2014.

[4] J. Maldacena, "The large $N$ limit of superconformal field theories and supergravity," Advances in Theoretical and Mathematical Physics, vol. 2, no. 2, pp. 231-252, 1998.

[5] S. S. Gubser, I. R. Klebanov, and A. M. Polyakov, "Gauge theory correlators from non-critical string theory," Physics Letters. B, vol. 428, no. 1-2, pp. 105-114, 1998.

[6] E. Witten, "Anti de sitter space and holography," Advances in Theoretical and Mathematical Physics, vol. 2, Article ID 9802150, pp. 253-291, 1998, http://arxiv.org/abs/hep-th/9802150.

[7] O. Aharony, S. S. Gubser, J. Maldacena, H. Ooguri, and Y. Oz, "Large $N$ field tTheories, string theory and Ggravity," Physics Reports, vol. 323, no. 3-4, pp. 183-386, 2000.

[8] V. E. Hubeny, M. Rangamani, and T. Takayanagi, "A covariant holographic entanglement entropy proposal," Journal of High Energy Physics, vol. 2007, no. 7, article 62, 2007.

[9] M. Alishahiha, A. F. Astaneh, and M. R. M. Mozaffar, "Holographic entanglement entropy for 4D conformal gravity," Journal of High Energy Physics, vol. 2014, article 8, 2014.

[10] M. Alishahiha, A. F. Astaneh, and M. R. M. Mozaffar, "Entanglement entropy for logarithmic conformal field theory," Physical Review D, vol. 89, Article ID 065023, 2014.

[11] B. Chen and J.-J. Zhang, "Note on generalized gravitational entropy in Lovelock gravity," Journal of High Energy Physics, vol. 2013, article 185, 2013.

[12] S. S. Pal, "Extremal surfaces and entanglement entropy," Nuclear Physics B, vol. 882, pp. 352-385, 2014.

[13] J. Erdmenger, M. Flory, and C. Sleight, "Conditions on holographic entangling surfaces in higher curvature gravity," Journal of High Energy Physics, vol. 2014, article 104, 2014.

[14] A. Bhattacharyya and M. Sharma, "On entanglement entropy functionals in higher-derivative gravity theories," Journal of High Energy Physics, vol. 2014, article 130, 2014.

[15] R. C. Myers and A. Singh, "Comments on holographic entanglement entropy and RG flows," Journal of High Energy Physics, vol. 2012, no. 4, article 122, 2012.

[16] N. Engelhardt and A. C. Wall, "Extremal surface barriers," Journal of High Energy Physics, vol. 2014, article 68, 2014.

[17] V. E. Hubeny, "Extremal surfaces as bulk probes in AdS/CFT," Journal of High Energy Physics, vol. 2012, article 93, 2012.

[18] X. Dong, "Holographic entanglement entropy for general higher derivative gravity," Journal of High Energy Physics, vol. 2014, p. 44, 2014.

[19] J. Camps, "Generalized entropy and higher derivative gravity," Journal of High Energy Physics, vol. 2014, no. 3, article 70, 2014.

[20] D. V. Fursaev, A. Patrushev, and S. N. Solodukhin, "Distributional geometry of squashed cones," Physical Review D, vol. 88, no. 4, Article ID 044054, 13 pages, 2013.

[21] D. V. Fursaev, "Proof of the holographic formula for entanglement entropy," Journal of High Energy Physics, no. 9, article 018, 2006.

[22] J. de Boer, M. Kulaxizi, and A. Parnachev, "Holographic entanglement entropy in Lovelock gravities," Journal of High Energy Physics, vol. 2011, article 109, 2011.

[23] L.-Y. Hung, R. C. Myers, and M. Smolkin, "On holographic entanglement entropy and higher curvature gravity," Journal of High Energy Physics, vol. 2011, article 25, 2011. 
[24] A. Buchel, J. Escobedo, R. C. Myers, M. F. Paulos, A. Sinha, and M. Smolkin, "Holographic GB gravity in arbitrary dimensions," Journal of High Energy Physics, vol. 2010, article 111, 2010.

[25] X. O. Camanho and J. D. Edelstein, "Causality in AdS/CFT and Lovelock theory," Journal of High Energy Physics, vol. 2010, p. 99, 2010.

[26] S. S. Pal, "Fermi-like liquid from Einstein-DBI-dilaton system," Journal of High Energy Physics, vol. 2013, article 7, 2013.

[27] I. R. Klebanov, D. Kutasov, and A. Murugan, "Entanglement as a probe of confinement," Nuclear Physics, B, vol. 796, no. 1-2, pp. 274-293, 2008.

[28] T. Jacobson and R. C. Myers, "Black hole entropy and highercurvature interactions," Physical Review Letters, vol. 70, no. 24, pp. 3684-3687, 1993.

[29] R.-G. Cai, “Gauss-Bonnet black holes in AdS spaces," Physical Review D, vol. 65, no. 8, Article ID 084014, 2002.

[30] I. Nojiri and S. D. Odintsov, "Anti-de-Sitter black hole thermodynamics in higher derivative gravity and new confiningdeconfining phases in dual CFT," Physics Letters B, vol. 521, no. 1-2, pp. 87-95, 2001, Erratum in Physics Letters B, vol. 542, p. 301, 2002.

[31] M. Cvetič, S. Nojiri, and S. D. Odintsov, "Black hole thermodynamics and negative entropy in de SITter and anti-de SITter Einstein-Gauss-Bonnet gravity," Nuclear Physics B, vol. 628, no. 1-2, pp. 295-330, 2002.

[32] Y. M. Cho and I. P. Neupane, "Anti-de Sitter black holes, thermal phase transition, and holography in higher curvature gravity," Physical Review D, vol. 67, Article ID 024044, 2002, I. P. Neupane, Phys. Rev. D 67, 061501 (2003), [hep-th/0212092].

[33] I. P. Neupane, "Black hole entropy in string-generated gravity models," Physical Review D: Particles, Fields, Gravitation and Cosmology, vol. 67, Article ID 061501, 2003.

[34] I. P. Neupane, "Thermodynamic and gravitational instability on hyperbolic spaces," Physical Review D, vol. 69, no. 8, Article ID 084011, 18 pages, 2004.

[35] M. Headrick and T. Takayanagi, "Holographic proof of the strong subadditivity of entanglement entropy," Physical Review D. Particles, Fields, Gravitation, and Cosmology, vol. 76, no. 10, Article ID 106013, 2007.

[36] A. Buchel and R. C. Myers, "Causality of holographic hydrodynamics," Journal of High Energy Physics, vol. 2009, no. 8, article 16, 2009.

[37] X.-H. Ge and S.-J. Sin, "Shear viscosity, instability and the upper bound of the Gauss-Bonnet coupling constant," Journal of High Energy Physics, vol. 2009, no. 5, article 51, 2009.

[38] X.-H. Ge, S.-J. Sin, S.-F. Wu, and G.-H. Yang, "Shear viscosity and instability from third order Lovelock gravity," Physical Review D, vol. 80, Article ID 104019, 2009.

[39] X.-H. Ge, Y. Matsuo, F.-W. Shu, S.-J. Sin, and T. Tsukioka, "Viscosity bound, causality violation and instability with stringy correction and charge," Journal of High Energy Physics, vol. 2008, no. 10, p. 009, 2008. 

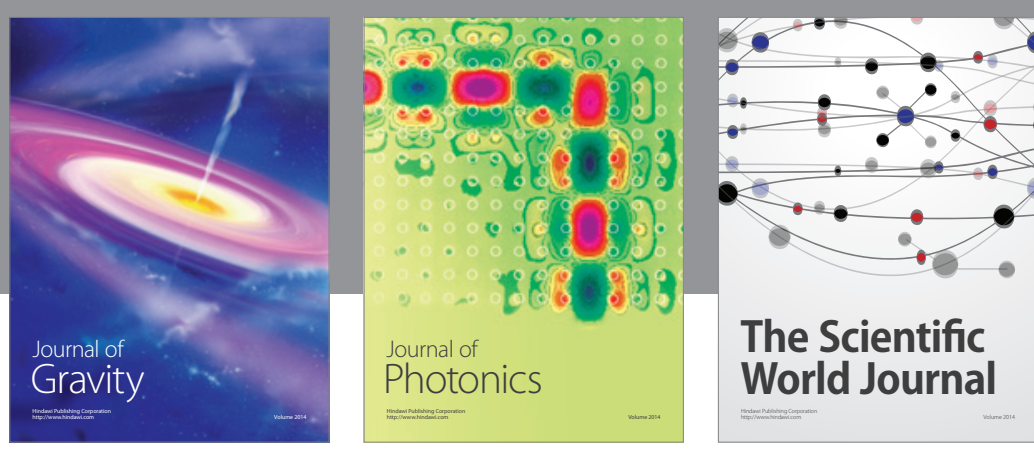

The Scientific World Journal
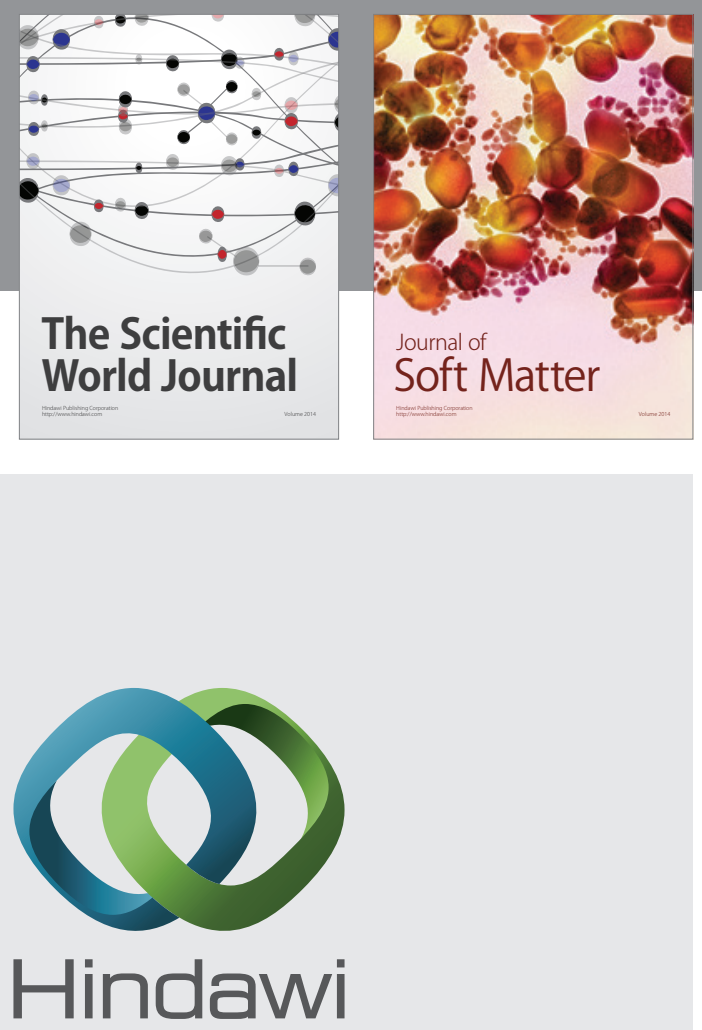

Submit your manuscripts at

http://www.hindawi.com

nternational Journal of

Statistical Mechanics
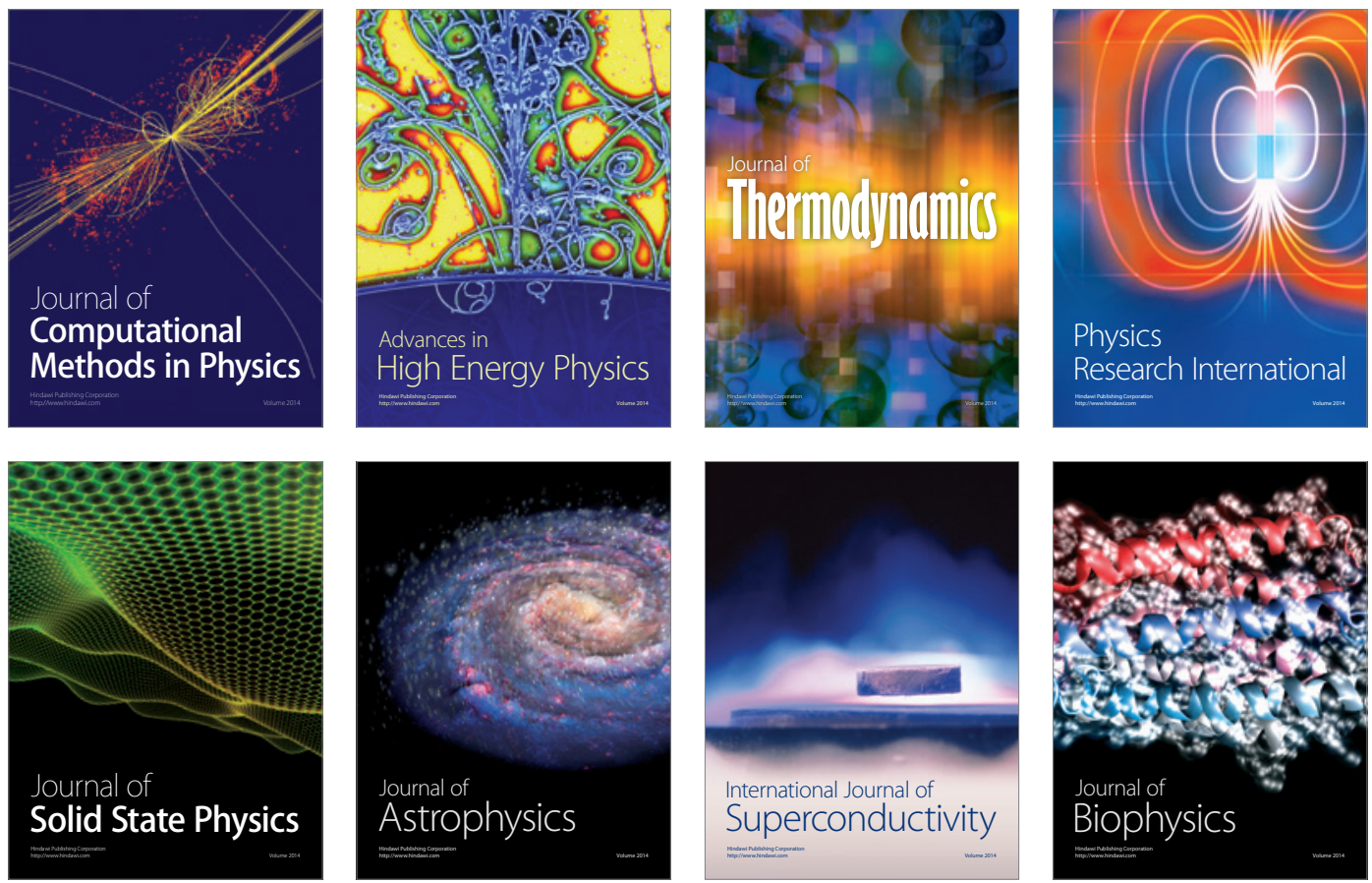
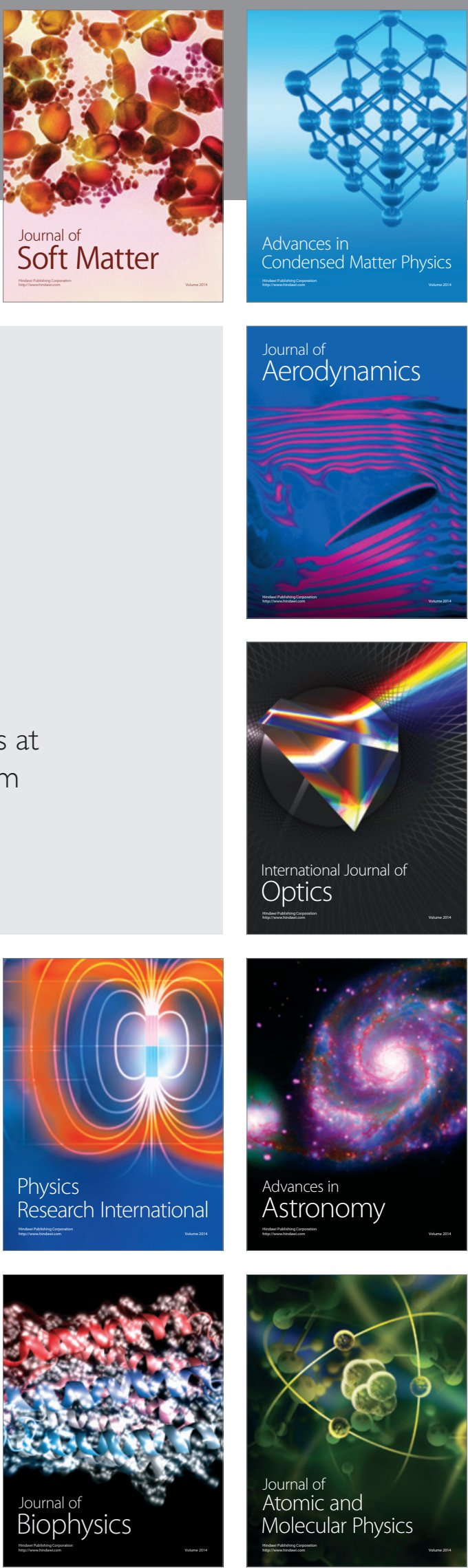